\title{
Measuring the mutual effects between a CZT detector and MRI for the development of a simultaneous $\mathrm{MBI} / \mathrm{MRI}$ insert
}

\author{
Ashley Tao ${ }^{1 *}$, Troy Farncombe ${ }^{2}$, Michael Noseworthy ${ }^{1}$ \\ From PSMR 2015: 4th Conference on PET/MR and SPECT/MR \\ La Biodola, Isola d'Elba, Italy. 17-21 May 2015
}

${ }^{1}$ McMaster University, Canada

While mammography is the gold standard for breast cancer screening, it suffers from poor sensitivity in women with dense breast tissue. Both breast MRI and molecular breast imaging (MBI) have been used as secondary imaging techniques. However, breast MRI suffers from low specificity and low sensitivity in MBI. A CZT based detector system has been developed with the goal of simultaneous MBI/MRI imaging to address the shortcomings of each modality. The performance of each modality needs to be addressed separately and together. The CZT system is comprised of four Redlen CZT modules tiled in a 2x2 array. Each module consists of 256 pixels and feature a builtin on-board ASIC and FPGA. A custom digital readout circuit board was designed to interface the four modules with a microcontroller to a PC. MR images were acquired with a 3T GE Discovery MR750 and Hologic breast coils. A gradient echo imaging sequence was used for all image acquisitions. A tissue mimicking phantom with a plastic grid insert $(1 \mathrm{~cm}$ spacing) was used to evaluate geometric accuracy with the CZT detectors in the MRI bore. The average distance between the grid markers was $1 \AA$ $\} 0.2 \mathrm{~cm}$ indicating negligible geometric distortion. Field maps were generated with a uniform phantom to quantify the effect on magnetic field homogeneity. Early results indicate a significant distortion $(\sim 10 \mathrm{ppm})$ in the magnetic field closest to the coil. Further analysis of the MR images will determine the extent of image quality degradation. A flood map of Tc-99m was acquired to evaluate and implement an energy correction map and a uniformity map. In the absence of a magnetic field, the mean energy resolution at $140 \mathrm{keV}$ was $6.3 \%$. After fully characterizing the uniformity, geometric accuracy and sensitivity, the same metrics will be evaluated in the MRI bore.

Authors' details

${ }^{1}$ McMaster University, Canada. ${ }^{2}$ Hamilton Health Sciences, Canada.

Published: 18 May 2015 